\title{
A study of second order impulsive neutral evolution differential control systems with an infinite delay
}

\author{
P. Palani, T. Gunasekar $†$ M. Angayarkanni ${ }^{\ddagger}$ and D. Kesavan $§$
}

\begin{abstract}
This article, we study the sufficient conditions for the controllability of second-order impulsive partial neutral evolution differential systems with infinite delay in Banach spaces by using the theory of cosine families of bounded linear operators and fixed point theorem.
\end{abstract}

Keywords: Nonlinear ordinary differential operators, Impulsive optimal control problems; Evolution equations.

2000 Subject Classification: 34L30, 49N25, 37L05.

\section{Introduction}

The study of impulsive functional differential equations is related to their utility in simulating processes and phenomena subject to short-time perturbations during their evolution. The perturbations are executed discretely and their duration is negligible in comparison with the total duration of the processes. That is why the perturbations are considered to take place instantaneously in the form of impulses. The theory of impulsive systems provides a common frame work for mathematical modeling of many real world phenomena. Moreover, these impulsive phenomena can also be found in fields such as information science, electronics, fed-batch culture in fermentative production, robotics and telecommunications (see [1, 16, [5, 14, 12, 17] and references therein).

${ }^{*}$ Sri Vidya Mandir Arts \& Science College, Uthangarai, Krishnagiri - 635307, Tamil Nadu, India. Email: ppalanitamil@gmail.com

${ }^{\dagger}$ Corresponding Author: Department of Mathematics, Vel Tech Rangarajan Dr.Sagunthala R\&D Institute of Science and Technology, Chennai - 600062, Tamil Nadu, India. Email: tguna84@gmail.com

${ }^{\ddagger}$ Department of Mathematics, Kandasamy Kandars College, P.Vellore, Namakkal-638182, Tamil Nadu, India. Email: angarkanni66@rediffmail.com

${ }^{\S}$ Department of Mathematics, Padmavani Arts \& Science College for Women, Salem - 636011, Tamil Nadu, INDIA. email: kesavan989@gmail.com 
In recent years, the study of impulsive control systems has received increasing interest. Due to its importance several authors have investigated the controllability of impulsive systems (see [2, 6]).

Motivated by the effort of the after mentioned papers [2, 9], the primary inspiration driving this manuscript is mainly concerned with the study of controllability of second order impulsive partial neutral system of the form

$$
\begin{aligned}
& \frac{d}{d \grave{u}}\left[\vartheta^{\prime}(\grave{u})-\theta_{1}\left(\grave{u}, \vartheta_{\grave{u}}\right)\right]=\mathbb{A}(\grave{u}) \vartheta(\grave{u})+B u(\grave{u})+\theta_{2}\left(\grave{u}, \vartheta_{\grave{u}}\right), \grave{u} \in J=[0, a], \grave{u} \neq \grave{u}_{k}, k=1,2, \ldots, n \\
& \vartheta_{0}=\phi \in \mathcal{B}, \quad \vartheta^{\prime}(0)=\xi \in X \\
& \Delta \vartheta\left(\grave{u}_{k}\right)=I_{k}\left(\vartheta_{\grave{u}_{k}}\right), \quad k=1,2, \ldots, m \\
& \Delta \vartheta^{\prime}\left(\grave{u}_{k}\right)=J_{k}\left(\vartheta_{\grave{u}_{k}}\right), \quad k=1,2, \ldots, m
\end{aligned}
$$

where $\phi \in \mathcal{B}$ and $\zeta \in X$. The control function $u(\cdot)$ is given in $L^{2}(J, \mathbb{U})$, a Banach space of admissible control functions with $\mathbb{U}$ as a Banach space and $B: \mathbb{U} \rightarrow X$ as a bounded linear operator; For $\grave{u} \in J, x_{\grave{u}}$ represents the function $\vartheta_{\grave{u}}:(-\infty, 0] \rightarrow X$ defined by $\vartheta_{\grave{u}}(\theta)=$ $\vartheta(\grave{u}+\theta),-\infty<\theta \leq 0$ which belongs to some abstract phase space $\mathcal{B}$ defined axiomatically, $\theta_{1}, \theta_{2}: J \times \mathcal{B} \rightarrow X, I_{k}: \mathcal{B} \rightarrow X, J_{k}: \mathcal{B} \rightarrow X$ are appropriate functions and will be specified later. $0<\grave{u}_{1}<\ldots<\grave{u}_{n}<a$ are fixed numbers and the symbol $\Delta \xi(\grave{u})$ represents the jump of a function $\xi$ at $\grave{u}$, which is defined by $\Delta \xi(\grave{u})=\xi\left(\grave{u}^{+}\right)-\xi\left(\grave{u}^{-}\right)$. Throughout the text we will assume that $\mathbb{A}(\cdot)$ generates an evolution operator $\mathbb{S}(\grave{u}, s)$.

\section{Preliminaries}

The theoretical non-autonomous second order initial value problem

$$
\begin{aligned}
& \vartheta^{\prime \prime}(\grave{u})=\mathbb{A}(\grave{u}) \vartheta(\grave{u})+f(\grave{u}), \quad 0 \leq s, \grave{u} \leq a, \\
& \vartheta(s)=v, \quad \vartheta^{\prime}(s)=w
\end{aligned}
$$

where, $\mathbb{A}(\grave{u}): D(\mathbb{A}(\grave{u})) \subseteq \vartheta \rightarrow X, \grave{u} \in J=[0, a]$ is a closed densely defined operator and $f: J \rightarrow X$ is an suitable function. Equations of this form have been considered in several papers. We refer the reader to [13, 15] and the references therein. In the majority of works, the existence of results to the problem (5)-(6) is related to the existence of an evolution operator $S(\grave{u}, s)$ for the homogeneous equation,

$$
\vartheta^{\prime \prime}(\grave{u})=\mathbb{A}(\grave{u}) \vartheta(\grave{u}), \quad 0 \leq s, \grave{u} \leq a .
$$

Let as assume that the domain of $\mathbb{A}(\grave{u})$ is a subspace of $D$ dense in $X$ and not dependent of $\grave{u}$, and for each $\vartheta \in D$ the function $\grave{u} \longmapsto \mathbb{A}(\grave{u}) \vartheta$ is continuous. The fundamental solution for the second-order evolution equation (7), has been developed by Kozak [11, and we will 
use the following concept of evolution operator.

Definition 2.1 [16] A family $\mathbb{S}$ of a bounded linear operator $\mathbb{S}(\grave{u}, s): J \times J \rightarrow L(\vartheta)$ is called an evolution operator for (7), if the following conditions are satisfied:

$(\mathbb{Z} 1)$ For each $\vartheta \in X$, the mappings $(\grave{u}, s) \in[0, a] \times[0, a] \rightarrow \mathbb{S}(\grave{u}, s) \vartheta \in X$ of class $\mathbb{C}^{1}$ and

(i) For each $\grave{u} \in[0, a], \mathbb{S}(\grave{u}, \grave{u})=0$,

(ii) For all $\grave{u}, s \in[0, a]$, and for each $\vartheta \in X$,

$$
\left.\frac{\partial}{\partial \grave{u}} \mathbb{S}(\grave{u}, s) \vartheta\right|_{\grave{u}=s}=\vartheta,\left.\quad \frac{\partial}{\partial s} \mathbb{S}(\grave{u}, s) \vartheta\right|_{\grave{u}=s}=-\vartheta
$$

$(\mathbb{Z} 2)$ For all $\grave{u}, s \in[0, a]$ if $\vartheta \in D(\mathbb{A})$, then $\mathbb{S}(\grave{u}, s) \vartheta \in D(\mathbb{A})$, the mappings $(\grave{u}, s) \in[0, a] \times$ $[0, a] \rightarrow \mathbb{S}(\grave{u}, s) \vartheta \in \vartheta$ is of class $\mathbb{C}^{2}$ and

(i) $\frac{\partial^{2}}{\partial \grave{u}^{2}} \mathbb{S}(\grave{u}, s) \vartheta=\mathbb{A}(\grave{u}) \mathbb{S}(\grave{u}, s) \vartheta$,

(ii) $\frac{\partial^{2}}{\partial s^{2}} \mathbb{S}(\grave{u}, s) \vartheta=\mathbb{S}(\grave{u}, s) \mathbb{A}(s) \vartheta$,

(iii) $\left.\frac{\partial}{\partial s} \frac{\partial}{\partial \grave{u}} \mathbb{S}(\grave{u}, s) \vartheta\right|_{\grave{u}=s}=0$.

$(\mathbb{Z} 3)$ For all $\grave{u}, s \in[0, a]$ if $\vartheta \in D(\mathbb{A})$, then $\frac{\partial}{\partial s} \mathbb{S}(\grave{u}, s) \vartheta \in D(\mathbb{A})$, there exists $\frac{\partial^{2}}{\partial \grave{u}^{2}} \frac{\partial}{\partial s} \mathbb{S}(\grave{u}, s) \vartheta, \frac{\partial^{2}}{\partial s^{2}} \frac{\partial}{\partial \grave{u}^{2}} \mathbb{S}(\grave{u}, s) \vartheta$ and

(i) $\frac{\partial^{2}}{\partial \grave{u}^{2}} \frac{\partial}{\partial s} \mathbb{S}(\grave{u}, s) \vartheta=\mathbb{A}(\grave{u}) \frac{\partial}{\partial s} \mathbb{S}(\grave{u}, s) \vartheta$,

(ii) $\frac{\partial^{2}}{\partial s^{2}} \frac{\partial}{\partial \grave{u}} \mathbb{S}(\grave{u}, s) \vartheta=\frac{\partial}{\partial \grave{u}} \mathbb{S}(\grave{u}, s) \mathbb{A}(s) \vartheta$ and the mapping $[0, a] \times[0, a] \ni(\grave{u}, s) \rightarrow \mathbb{A}(\grave{u}) \frac{\partial}{\partial s} \mathbb{S}(\grave{u}, s) \vartheta$ is continuous.

Throughout this problem [2, 16] we assume that there exists an evolution operator $\mathbb{S}(\grave{u}, s)$ associated to the operator $\mathbb{A}(\grave{u})$. To abbreviate the text, we introduce the operator $\mathbb{C}(\grave{u}, s)=$ $-\frac{\partial \mathbb{S}(\grave{u}, s)}{\partial s}$. In addition, we set $\mathbb{N}$ and $\widetilde{\mathbb{N}}$ for positive constants such that $\sup _{0<s, \grave{u}<a}\|\mathbb{S}(\grave{u}, s)\| \leq$ $\mathbb{N}$ and $\sup _{0<s, \grave{u}<a}\|\mathbb{C}(\grave{u}, s)\| \leq \widetilde{\mathbb{N}}$. In addition, we denote by $\mathbb{N} 1$ is a positive constant such that,

$$
\|\mathbb{S}(\grave{u}+h, s)-\mathbb{S}(\grave{u}, s)\| \leq \mathbb{N}_{1}|h|,
$$

for all $s, \grave{u}, \grave{u}+h \in[0, a]$. Assuming that $f: J \rightarrow X$ is an integrable function, the mild solution $\vartheta:[0, a] \rightarrow X$ of the problem (5)-(6) is given by,

$$
\vartheta(\grave{u})=\mathbb{C}(\grave{u}, s) v+\mathbb{S}(\grave{u}, s) w+\int_{s}^{\grave{u}} \mathbb{S}(\grave{u}, \tau) f(\tau) d \tau .
$$

In the literature a number of methods have been discussed to establish the existence of the evolution operators $\mathbb{S}(.,$.$) and \mathbb{C}(.,$.$) . In particular, a very studied situation is that \mathbb{A}(\grave{u})$ is that perturbation of an operator $\mathbb{A}$ that generates a cosine operator function. In this reason, below we briefly analysis some essential properties of the theory of cosine functions. Let 
$\mathbb{A}: D(\mathbb{A}) \subseteq \vartheta \rightarrow X$ be the infinitesimal generator of a strongly continuous cosine family of bounded linear operators $(\mathbb{C}(\grave{u}))_{\grave{u} \in R}$ on Banach space $\vartheta$. We denote by $(\mathbb{S}(\grave{u}))_{\grave{u} \in R}$ the sine function associated with $(\mathbb{C}(\grave{u}))_{\grave{u} \in R}$ which is defined by $\mathbb{S}(\grave{u}) \vartheta=\int_{0}^{\grave{u}} \mathbb{C}(s) \vartheta d s$, for $\vartheta \in X$ and $\grave{u} \in R$. We refer them to [3, 19] for the necessary concepts about cosine functions. After that we only mention a few results and notations about this matter needed to establish our results. It is immediate that

$$
\mathbb{C}(\grave{u}) \vartheta-\vartheta=\mathbb{A} \int_{0}^{\grave{u}} \mathbb{S}(s) \vartheta d s
$$

for all $\vartheta \in X$. The notation $D(\mathbb{A})$ stands for the domain of the operator $\mathbb{A}$ endowed with the graph norm $\|\vartheta\|_{\mathbb{A}}=\|\vartheta\|+\|\mathbb{A} \vartheta\|, \vartheta \in D(\mathbb{A})$. Moreover, in this work, $E$ is the space formed by the vectors $\vartheta \in \vartheta$ for which $\mathbb{C}(.) \vartheta$ is of class $\mathbb{C}^{1}$ on $R$. It was proved by Kisynski [10] that $E$ endowed with the norm $\|\vartheta\|_{E}=\|\vartheta\|+\sup _{0 \leq t \leq 1}\|\mathbb{A} \mathbb{S}(t) \vartheta\|, \vartheta \in E$, is a Banach space. The operator-valued function

$$
\mathcal{H}(\grave{u})=\left[\begin{array}{ll}
\mathbb{C}(\grave{u}) & \mathbb{S}(\grave{u}) \\
\mathbb{A} \mathbb{S}(\grave{u}) & \mathbb{C}(\grave{u})
\end{array}\right]
$$

is a strongly continuous group of bounded linear operators on the space $E \times \vartheta$ generated by the operator $\mathcal{H}(\grave{u})=\left[\begin{array}{ll}0 & 1 \\ \mathbb{A} & 0\end{array}\right]$ defined on $D(\mathbb{A}) \times E$. From this, it follows that $\mathbb{S}(\grave{u}): X \rightarrow E$ is a bounded linear map such that the operator valued maps $\mathbb{S}($.$) is strongly continuous and$ $\mathbb{A} \mathbb{S}(\grave{u}): E \rightarrow X$ is a bounded linear operator such that $\mathbb{A}(\mathbb{S}) \vartheta \rightarrow 0$ as $\grave{u} \rightarrow 0$, for each $\vartheta \in E$. Furthermore, if $\vartheta:[0, \infty) \rightarrow X$ is a locally integrable function, then the function $y(\grave{u})=\int_{0}^{\grave{u}} \mathbb{S}(\grave{u}-s) \vartheta(s) d s$ defines an $E$-valued continuous function.

The existence of solutions for the second order abstract Cauchy problem,

$$
\begin{aligned}
& \vartheta^{\prime \prime}(\grave{u})=\mathbb{A} \vartheta(\grave{u})+h(\grave{u}), \quad 0 \leq \grave{u} \leq a \\
& \vartheta(s)=v, \vartheta^{\prime}(s)=\omega
\end{aligned}
$$

where $h: J \rightarrow X$ is an integrable function, has been discussed in [20]. Similarly the existence of solutions of semilinear second order abstract Cauchy problems has been treated in [21]. We only mention here that the function $\vartheta(\cdot)$ given by

$$
\vartheta(\grave{u})=\mathbb{C}(\grave{u}-s) v+\mathbb{S}(\grave{u}-s) \omega+\int_{s}^{\grave{u}} \mathbb{S}(\grave{u}-\tau) h(\tau) d \tau, \quad 0 \leq \grave{u} \leq a,
$$

is called a mild solution of (7)-(8) and that when $v \in E, \vartheta($.$) is continuously differentiable$ and

$$
\vartheta^{\prime}(\grave{u})=\mathbb{A} \mathbb{S}(\grave{u}-s) v+\mathbb{C}(\grave{u}-s) \omega+\int_{s}^{\grave{u}} \mathbb{C}(\grave{u}-\tau) h(\tau) d \tau \quad 0 \leq \grave{u} \leq a .
$$


In addition, if $v \in D(\mathbb{A}), \omega \in E$ and $f$ is a continuously differentiable function, then the function $\vartheta(\cdot)$ is a solution of the IVP (11)-(12).

Assume that $\mathbb{A}(\grave{u})=\mathbb{A}+\widetilde{B}(\grave{u})$ where $\widetilde{B}(\cdot): R \rightarrow \mathcal{L}(E, \vartheta)$ is a map such that the function $\grave{u} \mapsto \widetilde{B}(\grave{u}) \vartheta$ is continuously differentiable in $\vartheta$, for each $\vartheta \in E$. It has been established by serizawa [18] that for each $(v, \omega) \in D(\mathbb{A}) \times E$ the non-autonomous abstract Cauchy problem

$$
\begin{aligned}
\vartheta^{\prime \prime}(\grave{u}) & =(\mathbb{A}+\widetilde{B}(\grave{u})) \vartheta(\grave{u}), \grave{u} \in R, \\
\vartheta(0) & =v, \quad \vartheta^{\prime}(0)=\omega
\end{aligned}
$$

has a unique solution $\vartheta(\cdot)$ such that the function $\grave{u} \mapsto \vartheta(\grave{u})$ is continuously differentiable in $E$. It is clear that the same argument allows us to conclude that Eq.(14), with the initial condition (12) has a unique solution $\vartheta(\cdot, s)$ such that the function $\grave{u} \mapsto \vartheta(\grave{u}, s)$ is continuously differentiable in $E$. It follows from (13) that

$$
\vartheta(\grave{u}, s)=\mathbb{C}(\grave{u}-s) v+\mathbb{S}(\grave{u}-s) \omega+\int_{s}^{\grave{u}} \mathbb{S}(\grave{u}-\tau) \widetilde{B}(\tau) \vartheta(\tau, s) d \tau
$$

In particular, for $v=0$ we have

$$
\vartheta(\grave{u}, s)=\mathbb{S}(\grave{u}-s) \omega+\int_{s}^{\grave{u}} \mathbb{S}(\grave{u}-\tau) \widetilde{B}(\tau) \vartheta(\tau, s) d \tau .
$$

Consequently,

$$
\|\vartheta(\grave{u}, s)\|_{1} \leq\|\mathbb{S}(\grave{u}-s)\|_{\mathcal{L}(\vartheta, E)}\|\omega\|+\int_{s}^{\grave{u}}\|\mathbb{S}(\grave{u}, \tau)\|_{\mathcal{L}(\vartheta, E)}\|\widetilde{B}(\tau)\|_{\mathcal{L}(\vartheta, E)} \| \vartheta\left(\tau, s \|_{1} d \tau\right.
$$

and applying the Gronwall - Bellman lemma we have,

$$
\|\vartheta(\grave{u}, s)\|_{1} \leq \widetilde{M}\|\omega\|, \quad s, \grave{u} \in J
$$

We define the operator $\mathbb{S}(\grave{u}, s) \omega=\vartheta(\grave{u}, s)$. It follows from the previous estimate that $\mathbb{S}(\grave{u}, s)$ is a bounded linear map on $E$. Since $E$ is dense in $X$, we can extend $\mathbb{S}(\grave{u}, s)$ to $X$. We keep the notation $\mathbb{S}(\grave{u}, s)$ for this extension. It is well known that, exception the case $\operatorname{dim}(X)<\infty$, the cosine function $\mathbb{C}(\grave{u})$ cannot be compact for all $\grave{u} \in \mathbb{R}$. By contrast, for the cosine functions that arise in specific applications, the sine function $\mathbb{S}(\grave{u})$ is very often a compact operator for all $\grave{u} \in \mathbb{R}$. This motivates the result ([7], Theorem 1.2).

Here we introduced the space $\mathcal{P} \mathbb{C}$ formed by all normalized piece wise continuous functions $\vartheta:[0, a] \rightarrow X$ such that $\vartheta(\cdot)$ is continuous at $\grave{u} \neq \grave{u}_{k}, \vartheta\left(\grave{u}_{k^{-}}\right)=\vartheta\left(\grave{u}_{k}\right)$ and $\vartheta\left(\grave{u}_{k}^{+}\right)$exists, for $k=1,2, \ldots, m$. In this paper, we always assume that $\mathcal{P} \mathbb{C}$ is endowed with the norm $\|\vartheta\|_{\mathcal{P C}}=\sup _{s \in J}\|\vartheta(s)\|$. It is clear that $\left(\mathcal{P} \mathbb{C},\|\cdot\|_{\mathcal{P} \mathbb{C}}\right)$ is a Banach space.

It follows that, we put $\grave{u}_{0}=0, \grave{u}_{n+1}=a$ and for $\vartheta \in \mathcal{P} \mathbb{C}$, we denote by $\tilde{\vartheta}_{k}$, for $k=$ $0,1, \ldots, m$, the function $\tilde{\vartheta}_{k} \in \mathbb{C}\left(\left[\grave{u}_{k}, \grave{u}_{k+1}\right] ; \vartheta\right)$ given by $\tilde{\vartheta}_{k}(\grave{u})=\vartheta(\grave{u})$ for $\grave{u} \in\left(\grave{u}_{k}, \grave{u}_{k+1}\right)$ and 
$\tilde{\vartheta}_{k}\left(\grave{u}_{k}\right)=\lim _{\tilde{u}_{u} \rightarrow \grave{u}^{+}} \vartheta(\grave{u})$. Moreover, for a set $E \subseteq \mathcal{P} \mathbb{C}$, we denote by $\tilde{E}_{k}$, for $k=0,1, \ldots, m$, the set $\tilde{E}_{k}=\tilde{\vartheta}_{k}: \vartheta \in E$.

Lemma $2.1[16]$ ] $A$ set $E \subseteq \mathcal{P} \mathbb{C}$ is relatively compact in $\mathcal{P} \mathbb{C}$ if and only if each $\widetilde{E_{k}}, k=$ $0,1, \ldots, m$, is relatively compact in $\mathbb{C}\left(\left[\grave{u}_{k}, \grave{u}_{k+1}\right] ; \vartheta\right)$.

In this work we will employ an axiomatic definition of the phase space $\mathcal{B}$, similar to the one used in [8] and suitably modify to treat retarded impulsive differential equations. More precisely, $\mathcal{B}$ will denote the vector space of functions defined from $(-\infty, 0]$ into $\vartheta$ endowed with a seminorm denoted $\|\cdot\|_{\mathcal{B}}$ and such that the following axioms are hold:

(A) If $\vartheta:(-\infty, \mu+b] \rightarrow \vartheta, b>0$, is such that $\vartheta_{\mu} \in \mathcal{B}$ and $\left.\vartheta\right|_{[\mu, \mu+b]} \in \mathcal{P} \mathbb{C}([\mu, \mu+b], X)$ then, for every $\grave{u} \in[\mu, \mu+b)$, the following conditions are hold:and

(i) $\vartheta_{\grave{u}}$ is $\in \mathcal{B}$,

(ii) $\|\vartheta(\grave{u})\| \leq H\left\|\vartheta_{\grave{u}}\right\|_{\mathcal{B}}$

(iii) $\left\|\vartheta_{\grave{u}}\right\|_{\mathcal{B}} \leq K(\grave{u}-\mu) \sup \{\|\vartheta(s)\|: \mu \leq s \leq \grave{u}\}+M(\grave{u}-\mu)\left\|\vartheta_{\mu}\right\|_{\mathcal{B}}$,

where $H>0$ is a constant; $K, M:[0, \infty) \rightarrow[1, \infty), K$ is continuous, $M$ is locally bounded and $H, K, M$ are independent of $\vartheta(\cdot)$.

(B) The space $\mathcal{B}$ is complete.

Remark 2.1 In impulsive functional differential systems, the map $[\mu, \mu+b] \rightarrow \mathcal{B}, \grave{u} \rightarrow \vartheta_{t}$, is in general discontinuous. For this reason, this property has been omitted from our description of the phase space $\mathcal{B}$.

Now we include that some of our proofs are based on the following well-known result ([4], Theroem 6.5.4).

Lemma 2.2 ([2]) Let $D$ be a closed convex subsets of a normed lined space $\vartheta$ such that $0 \in D$. Let $F: D \rightarrow D$ be a completely continuous map. Then the set $\{\vartheta \in D: \vartheta=$ $\lambda F(\vartheta)$, for some $0<\lambda<1\}$ is unbounded or the map $F$ has a fixed point in $D$.

The terminology and notations are generally used in functional analysis. In particular, for Banach spaces $(Z,\|\cdot\|),\left(W,\|\cdot\|_{w}\right)$, the notation $\mathcal{L}(Z, W)$ stands for the Banach space of bounded linear operators from $Z$ into $W$ and we abbreviate to $\mathcal{L}(Z)$ whenever $Z=W$. By $\sigma(\mathbb{A})$ (respectively,$\rho(\mathbb{A})$ ) we denote the spectrum (respectively , the resolvent set)of a linear operator $\mathbb{A}$. Moreover $B_{r}(\vartheta, Z)$ denotes the closed ball with center at $\vartheta$ and radius $r>0$ in the space $Z$. 


\section{Controllability result}

Definition 3.2 A function $\vartheta:(-\infty, a] \rightarrow X$ is called a mild solution of the abstract Cauchy problem (1)-(4), if $\vartheta_{0}=\phi \in \mathcal{B},\left.\vartheta\right|_{J} \in \mathcal{P} \mathbb{C}$, the impulsive conditions $\Delta \vartheta\left(\grave{u}_{k}\right)=I_{k}\left(\vartheta_{\grave{u}_{k}}\right)$, $\Delta \vartheta^{\prime}\left(\grave{u}_{k}\right)=J_{k}\left(\vartheta_{\grave{u}_{k}}\right), k=1,2 \ldots, m$ are satisfied and the following integral equation

$$
\begin{aligned}
\vartheta(\grave{u}) & =\mathbb{C}(\grave{u}, 0) \phi(0)+\mathbb{S}(\grave{u}, 0)\left(\vartheta-\theta_{1}(0, \phi, 0)\right)+\int_{0}^{\grave{u}} \mathbb{C}(\grave{u}, s) \theta_{1}\left(s, \vartheta_{s}\right) d s \\
& +\int_{0}^{\grave{u}} \mathbb{S}(\grave{u}, s)\left[B u(s)+\theta_{2}\left(s, \vartheta_{s}\right)\right] d s+\sum_{0<\grave{u}_{k}<\grave{u}} \mathbb{C}\left(\grave{u}, \grave{u}_{k}\right) I_{k}\left(\vartheta_{\grave{u}_{k}}\right)+\sum_{0<\grave{u}_{k}<\grave{u}} \mathbb{S}\left(\grave{u}, \grave{u}_{k}\right) J_{k}\left(\vartheta_{\grave{u}_{k}}\right), \quad 0<\grave{u}<a .
\end{aligned}
$$

is verified.

In what follows the notation $g(a)$ stands for the space

$$
\theta_{1}(a)=\left\{y:(-\infty, a] \rightarrow \vartheta:\left.y\right|_{J} \in \mathcal{P} \mathbb{C}, y_{0}=0\right\}
$$

endowed with the sup norm. In addition, we denote by $\widetilde{\phi}:(-\infty, a] \rightarrow \vartheta$ the function defined by $\widetilde{\phi}_{0}=\phi$ and $\widetilde{\phi}(\grave{u})=\mathbb{C}(\grave{u}, 0) \phi(0)+\mathbb{S}(\grave{u}, 0) \zeta$, for $\grave{u} \geq 0$. Let $B_{r}=\{\vartheta \in X,\|\vartheta\| \leq r\}$ for some $r>0$.

In order to obtain the controllability result, we introduce the following assumptions:

(H1) The function $\theta_{1}: J \times B \rightarrow X X$ is continuous and there exist constants $L_{f}>0, \widetilde{L_{f}}>0$ for $\psi_{1}, \psi_{2} \in \mathcal{B}$, such that

$$
\left\|\theta_{1}\left(\grave{u}, \psi_{1}\right)-\theta_{1}\left(\grave{u}, \psi_{2}\right)\right\| \leq L_{\theta_{1}}\left\|\psi_{1}-\psi_{2}\right\|_{\mathcal{B}}
$$

and $\widetilde{L}_{\theta_{1}}=\sup _{\grave{u} \in J}\left\|\theta_{1}(\grave{u}, 0)\right\|$.

$(\mathrm{H} 2) B$ is a continuous operator from $\mathbb{U}$ to $X$ and the linear operator $W: L^{2}(J, \mathbb{U}) \rightarrow X$, defined by

$$
W u=\int_{0}^{a} \mathbb{S}(a, s) B u(s) d s
$$

has a bounded invertible operator $W^{-1}$ which takes values in $L^{2}(J, \mathbb{U}) / \operatorname{ker} W$ and there exist positive constant $M$ such that $\left\|B W^{-1}\right\| \leq M_{1}$.

(H3) The impulsive functions satisfy the following conditions:

(i) The maps $I_{k}: \mathcal{B} \rightarrow X, k=1,2, \ldots, m$ is continuous and there exist constants $L_{1}>0, \widetilde{L}_{1}>0$ for $\psi_{1}, \psi_{2} \in \mathcal{B}$ such that

$$
\left\|I_{k}\left(\psi_{1}\right)-I_{k}\left(\psi_{2}\right)\right\| \leq L_{I}\left\|\psi_{1}-\psi_{2}\right\|
$$

and $\widetilde{L}_{I}=\left\|I_{k}(0)\right\|$. 
(ii) The maps $J_{k}: \mathcal{B} \rightarrow X, k=1,2, \ldots, m$ is continuous and there exists constants $L_{J}>0, \widetilde{L}_{J}>0$ for $\psi_{1}, \psi_{2} \in \mathcal{B}$ such that

$$
\left\|J_{k}\left(\psi_{1}\right)-J_{k}\left(\psi_{2}\right)\right\| \leq L_{J}\left\|\psi_{1}-\psi_{2}\right\|
$$

and $\widetilde{L}_{J}=\left\|J_{k}(0)\right\|$.

(H4) The function $\theta_{2}: J \times \mathcal{B} \rightarrow X$ is completely continuous and there exists $L_{\theta_{2}}>0$ such that

$$
\left\|\theta_{2}\left(\grave{u}, \psi_{1}\right)-\theta_{2}\left(\grave{u}, \psi_{2}\right)\right\| \leq L_{\theta_{2}}\left\|\psi_{1}-\psi_{2}\right\|_{\mathcal{B}}, \grave{u} \in J, \psi_{1}, \psi_{2} \in \mathcal{B}
$$

From (H4), we have $\left\|\theta_{2}(t, \psi)\right\| \leq L_{\theta_{2}}\|\psi\|_{\mathcal{B}}+L_{1}$ where $L_{1}=\sup _{\grave{u} \in J}\left\|\theta_{2}(\grave{u}, 0)\right\|$.

$$
\begin{aligned}
& \text { Let } a \mathbb{N}\left[L_{\theta_{1}}\left(K_{a} r+c_{1}\right)+\widetilde{L}_{\theta_{1}}\right]+a \mathbb{N} \mathbb{A}_{0}+\sum_{k=1}^{m}\left(\widetilde{\mathbb{N}} L_{I}+\mathbb{N} L_{J}\right)\left[K_{a} r+\left\|\widetilde{\phi}_{\dot{u}_{k}}\right\|\right]+\sum_{k=1}^{m}\left(\widetilde{\mathbb{N}} \widetilde{L}_{I}+\right. \\
& \left.\mathbb{N} \widetilde{L}_{J}\right) \leq r, \text { for some } r>0 .
\end{aligned}
$$

(H6) $\quad$ Let $\mu=k_{a}\left(1+a \mathbb{N} M_{1}\right)\left[a \widetilde{\mathbb{N}} L_{g}+a \mathbb{N} L_{\theta_{1}}+\sum_{k=1}^{m}\left(\widetilde{\mathbb{N}} L_{I}+\mathbb{N} L_{J}\right)<1\right.$ be such that $0 \leq \mu<1$.

Definition 3.3 The system (14-17) is said to be controllable on the interval $J$, if for every $\vartheta_{0}=\phi \in \mathcal{B}, \vartheta^{\prime}(0)=\zeta$ and $z_{1} \in X$, there exists a control $u \in L^{2}(J, \mathbb{U})$ such that the mild solution $\vartheta(\cdot)$ of (1)-(4) satisfies $\vartheta(a)=z_{1}$

The following results is an immediate application of the contraction principle of Banach. To simplify the text, we denote $K_{a}=\sup _{0 \leq \grave{u} \leq a} K(\grave{u})$.

Since $\left\|\widetilde{\phi}_{k}\right\|_{\mathcal{B}}=\widetilde{\mathbb{N}}\|\phi(0)\|+\mathbb{N}\|\zeta\|+M\|\phi\|_{\mathcal{B}}<\infty, 0 \leq t \leq a$, we denote, $c_{1}=\sup _{0 \leq \grave{u} \leq a}\left\|\phi_{\grave{u}}\right\|_{\mathcal{B}}$ and $\left\|y_{\grave{u}}+\widetilde{\phi}_{\grave{u}}\right\| \leq K_{a}\left\|y_{\grave{u}}\right\|+\|\widetilde{\phi}\| \leq K_{a} r+c_{1}=\rho$.

Theorem 3.1 If the hypothesis (H1)-(H6) are satisfied, then the impulsive second order system (1)-(4) is controllable on $J$.

Proof. Using the assumption (H2), we define the control function

$$
\begin{aligned}
u(\grave{u}) & =W^{-1}\left[z_{1}-\mathbb{C}(a, 0) \phi(0)-\mathbb{S}(a, 0)\left[\zeta-\theta_{2}(0, \phi)\right]+\int_{0}^{a} \mathbb{C}(a, s) \theta_{2}\left(s, \vartheta_{s}\right) d s\right. \\
& \left.-\int_{0}^{a} \mathbb{S}(a, s) \theta_{1}\left(s, \vartheta_{s}\right) d s-\sum_{k=1}^{m} \mathbb{C}\left(a, \grave{u}_{k}\right) I_{k}\left(\vartheta_{\grave{u}_{k}}\right)-\sum_{k=1}^{m} \mathbb{S}\left(a, \grave{u}_{k}\right) J_{K}\left(\vartheta_{\grave{u}_{k}}\right)\right](\grave{u})
\end{aligned}
$$

we shall now show that when using this control the operator $\Gamma$ on the space $\theta_{2}(a)$ defined 
$\operatorname{by}(\Gamma y)_{0}=0$ and

$$
\begin{aligned}
(\Gamma y)(\grave{u}) & =\mathbb{S}(\grave{u}, 0)\left[\zeta-\theta_{2}(0, \phi)\right]-\int_{0}^{\grave{u}} \mathbb{C}(\grave{u}, s) \theta_{2}\left(s, y_{s}+\widetilde{\phi}_{s}\right) d s+\int_{0}^{\grave{u}} \mathbb{S}(\grave{u}, s) \theta_{1}\left(s, y_{s}+\widetilde{\phi}_{s}\right) d s \\
& +\int_{0}^{\grave{u}} \mathbb{S}(\grave{u}, \eta) B W^{-1}\left[z_{1}-\mathbb{C}(a, 0) \phi(0)-\mathbb{S}(a, 0)\left[\zeta-\theta_{2}(0, \phi)\right]+\int_{0}^{a} \mathbb{C}(a, s) \theta_{2}\left(s, y_{s}+\widetilde{\phi}_{s}\right) d s\right. \\
& \left.-\int_{0}^{\grave{u}} \mathbb{S}(a, s) \theta_{1}\left(s, y_{s}+\widetilde{\phi}_{s}\right) d s-\sum_{k=1}^{m} \mathbb{C}\left(a, \grave{u}_{k}\right) I_{k}\left(y_{\grave{u}_{k}}+\widetilde{\phi}_{\grave{u}_{k}}\right)-\sum_{k=1}^{m} \mathbb{S}\left(a, \grave{u}_{k}\right) J_{k}\left(y_{\grave{u}_{k}}+\widetilde{\phi}_{\grave{u}_{k}}\right)\right](\eta) d \eta \\
& +\sum_{0<\grave{u}_{k}}<\grave{u} \mathbb{C}\left(\grave{u}, \grave{u}_{k}\right) I_{k}\left(y_{\grave{u}_{k}}+\widetilde{\phi}_{\grave{u}_{k}}\right)+\sum_{0<\grave{u}_{k}<\grave{u}} \mathbb{S}\left(\grave{u}, \grave{u}_{k}\right) J_{k}\left(y_{\grave{u}_{k}}+\widetilde{\phi}_{\grave{u}_{k}}\right), \quad \grave{u} \in J,
\end{aligned}
$$

has a fixed point $\vartheta(\cdot)$. This fixed point is then a mild solution of the system (1)-(4). Clearly $(\Gamma \vartheta)(a)=z_{1}$ which means that the control $u$ steers the system from the initial state $\phi$ to $z_{1}$ in time $a$, provided we can obtain a fixed point of the operator $\Gamma$ which implies that the system is controllable. From the assumptions, it is easy to see that $\Gamma$ is well defined and continuous. For convenience let us take,

$$
\begin{aligned}
\|B u(s)\| & \leq M_{1}\left[\left\|z_{1}\right\|+\widetilde{\mathbb{N}}\|\phi(0)\|+\mathbb{N}\left[\|\zeta\|+L_{\theta_{2}}\|\phi\|+\widetilde{L_{\theta_{2}}}\right]+\widetilde{\mathbb{N}} a\left[L_{\theta_{2}}\left(K_{a} r+c_{1}\right) \widetilde{L_{\theta_{2}}}\right]\right. \\
& +a \mathbb{N}\left[L_{\theta_{1}}\left(K_{a} r+c_{1}\right)+\widetilde{L}_{\theta_{1}}\right]+\widetilde{\mathbb{N}} \sum_{k=1}^{m}\left[L_{I}\left(K_{a} r+\left\|\widetilde{\phi}_{\grave{u}_{k}}\right\|\right)+\widetilde{L}_{I}\right] \\
& +\mathbb{N} \sum_{k=1}^{m}\left[l_{\theta_{1}}\left(K_{a} r+\|\widetilde{\phi}\|\right)+\widetilde{L}_{J}\right]=\mathbb{A}_{0}
\end{aligned}
$$

First we show that $\Gamma$ maps $B_{r}\left(0, \theta_{2}(a)\right)$ into $B_{r}\left(0, \theta_{2}(a)\right)$. To this end, from the definition of the operator $\Gamma$ in (18) and our hypotheses, we obtain

$$
\begin{aligned}
\|(\Gamma y)(\grave{u})\| & \leq \mathbb{N}\left[\|\zeta\|+L_{\theta_{2}}\|\phi\|+\widetilde{L_{g}}\right]+\widetilde{\mathbb{N}} a\left[L_{\theta_{2}}\left(K_{a} r+c_{1}\right) \widetilde{L_{\theta_{2}}}\right] \\
& +a \mathbb{N}\left[L_{\theta_{1}}\left(k_{a} r+c_{1}\right)+\widetilde{L}_{\theta_{1}}\right]+a \mathbb{N} \mathbb{A}_{0}+\sum_{k=1}^{m}\left(\widetilde{\mathbb{N}} \widetilde{L}_{I}+\mathbb{N} \widetilde{L}_{J}\right)+\sum_{k=1}^{M}\left(\widetilde{\mathbb{N}} L_{J}\right)\left[K_{a} r+\left\|\widetilde{\phi}_{\grave{u}_{k}}\right\|\right] \\
& \leq r .
\end{aligned}
$$

for $y \in \theta_{2}(a)$ and $\grave{u} \in J$. Hence $\|\Gamma y\|_{a} \leq r$. Therefore, $\Gamma$ maps $B_{r}\left(0, \theta_{2}(a)\right)$ into itself. Now for $y, z \in B_{r}\left(0, \theta_{2}(a)\right)$, we have

$$
\begin{aligned}
\|(\Gamma y)(\grave{u})-(\Gamma z)(\grave{u})\| & \leq K_{a}\left(1+a \mathbb{N} M_{1}\right)\left[a \left(\widetilde{\mathbb{N}} L_{\theta_{2}}+a \mathbb{N} L_{\theta_{1}}+\sum+k=1^{m}\left(\left(\widetilde{\mathbb{N}} L_{I}+\mathbb{N} L_{J}\right)\right]\|y-z\|\right.\right. \\
& \leq \mu\|y-z\|_{a},
\end{aligned}
$$

Which implies that $\Gamma$ is a contraction on $B_{r}\left(0, \theta_{2}(a)\right)$. Hence by the Banach fixed point theorem, $\Gamma$ has a unique fixed point $y$ in $\theta_{2}(a)$. Defining $\vartheta(\grave{u})=y(\grave{u})+\widetilde{\phi}(\grave{u}),-\infty<\grave{u} \leq a$, we obtain that $\vartheta(\cdot)$ is a mild solution of the problem (1)-(4) and the proof is complete.

We use the below condition instead of (H1) to avoid the Lipschitz continuity of $\mathrm{f}$ used in 
Theorem 3.1.

(A1) The function $\theta_{1}: J \times \mathcal{B} \rightarrow X$ satisfies the following conditions:

(i) For each $\grave{u} \in J$, the function $\theta_{1}(\grave{u}, \cdot): \mathcal{B} \rightarrow X$ is continuous and the function $\grave{u} \rightarrow \theta_{1}\left(\grave{u}, \vartheta_{\grave{u}}\right)$ is strongly measurable.

(ii) There exist an integrable function $p: J \rightarrow[0, \infty)$ and a continuous non-decreasing function $\Omega:[0, \infty) \rightarrow(0, \infty)$ such that

$$
\left\|\theta_{1}(\grave{u}, \psi)\right\| \leq p(\grave{u}) \Omega\left(\|\psi\|_{B},(\grave{u}, \psi) \in J \times \mathcal{B}\right.
$$

Also, we have the following condition.

$$
\begin{aligned}
& {\left[\left\|z_{1}\right\|+\mathbb{N}\|\phi(0)\|+\widetilde{\mathbb{N}}\left[\|\zeta\|+L_{\theta_{2}}\|\phi\|+\widetilde{L_{\theta_{2}}}\right]+\mathbb{N} a\left(L_{\theta_{2}} \rho+\widetilde{L_{\theta_{2}}}\right)\right.} \\
& \left.\quad+\mathbb{N} \int_{0}^{a} p(s) \Omega(\rho) d s+\widetilde{\mathbb{N}} \sum_{k=1}^{m}\left(\alpha_{k}^{1}(\rho)+\alpha_{k}^{2}\right)+\mathbb{N} \sum_{k=1}^{m}\left(\beta_{k}^{1}(\rho)+\beta_{k}^{2}\right)\right]=M^{*}
\end{aligned}
$$

Theorem 3.2 Assume that $\theta_{1}$ verify condition (A1) and condition (A2),(H2) are satisfied. Also, the following conditions are hold:

(a) For every $\grave{u} \in J$ and every $r>0$, the set $\mathbb{U}(r, \grave{u})=\left\{\mathbb{S}(\grave{u}, s) \theta_{1}(s, \psi): s \in[0, a], \psi \in\right.$ $\left.B_{r}(0, \mathcal{B})\right\}$ is relatively compact in $X$.

(b) The maps $I_{k}, J_{k}: \mathcal{B} \rightarrow X$ are completely continuous and there exist positive constants $\alpha_{k}^{i}, \beta_{k}^{i}, i=1,2, k=1,2, \ldots, m$, such that $\left\|I_{k}(\psi)\right\| \leq \alpha_{k}^{1}\|\psi\|_{\mathcal{B}}+\alpha_{k}^{2}$ and $\left\|J_{k}(\psi)\right\| \leq \beta_{k}^{1}\|\psi\|_{\mathcal{B}}+\beta_{k}^{2}$, for all $\psi \in \mathcal{B}$.

(c) The constant $\mu=\tilde{\mathbb{N}} a K_{a} L_{g}+K_{a} \sum_{k=1}^{m}\left(\tilde{\mathbb{N}} \alpha_{k}^{1}+\mathbb{N} \beta_{k}^{1}\right)<1$ and $\int_{c}^{\infty} \frac{d s}{\Omega(s)}>\frac{K_{\alpha} \mathbb{N}}{1-\mu} \int_{0}^{a} p(s) d s$ where $c=\frac{1}{1-\mu}\left[\mathbb{N}\left[\|\zeta\|+L_{\theta_{2}}\|\phi\|+\widetilde{L_{\theta_{2}}}\right]+\tilde{\mathbb{N}} a K_{a} \widetilde{L_{\theta_{2}}}+a \mathbb{N} M_{1} M^{*}+c_{1}+K_{a} \sum_{k=1}^{m}\left(\tilde{\mathbb{N}} \alpha_{k}^{2}+\mathbb{N} \beta_{k}^{2}\right)\right]$. Then the (1)-(4) is controllable on $J$.

Proof. we define the map $\Gamma$ on the space $\theta_{2}(a)$ as in eq (18). To prove the controllability of the problem (1)-(4), we must show that the operator $\Gamma$ has a fixed point. This fixed point is then a mild solution of the system (1)-(4). From the assumptions, it is easy to see that $\Gamma$ is well defined and continuous.

In order to apply Lemma 2.2, we need to obtain a priori bound for the solutions of the integral equation $y=\lambda \Gamma(y), \lambda \in(0,1)$. To this end, let $y^{\lambda}$ be a solution of $\lambda \Gamma(y)=y$, $\lambda \in(0,1)$. Using the notation $\nu^{\lambda}=\sup _{0 \leq s \leq \grave{u}}\left\|y_{s}^{\lambda}+\tilde{\phi}_{s}\right\|_{\mathcal{B}} \leq K_{a}\left\|y^{\lambda}\right\|_{s}+\left\|\tilde{\phi}_{s}\right\|_{\mathcal{B}, a} \leq K_{a} r+c_{1}=\rho$, 
we observe that

$$
\begin{aligned}
\left\|y^{\lambda}(\grave{u})\right\| & \leq \mathbb{N}\left[\|\zeta\|+L_{\theta_{2}}\|\phi\|+\widetilde{L_{\theta_{2}}}\right]+\tilde{\mathbb{N}} a K_{a}\left(L_{\theta_{2}} v^{\lambda}(s)+\widetilde{L_{\theta_{2}}}\right)+\mathbb{N} \int_{0}^{a} p(s) \Omega\left(v^{\lambda}(s)\right) d s \\
& +a \mathbb{N} M_{1}\left[M^{*}\right]+\sum_{0<\grave{u}_{k}<t}\left(\widetilde{\mathbb{N}} \alpha_{k}^{1}+\mathbb{N} \beta_{k}^{1}\right) v^{\lambda}\left(\grave{u}_{k}\right)+\sum_{0<\grave{u}_{k}<t}\left(\widetilde{\mathbb{N}} \alpha_{k}^{2}+\mathbb{N} \beta_{k}^{2}\right)
\end{aligned}
$$

Hence follows that

$$
\begin{aligned}
v^{\lambda}(\grave{u}) & \leq \mathbb{N}\left[\|\zeta\|+L_{\theta_{2}}\|\phi\|+\widetilde{L_{\theta_{2}}}\right]+\tilde{\mathbb{N}} a K_{a} \widetilde{L_{\theta_{2}}}+K_{a} \mathbb{N} \int_{0}^{t} p(s) \Omega\left(v^{\lambda}(s)\right) d s+a \mathbb{N} M_{1} M^{*}+\sup _{0 \leq s \leq t}\left\|\phi_{s}\right\|_{\mathcal{B}} \\
& +K_{a} \sum_{k=1}^{m}\left(\widetilde{\mathbb{N}} \alpha_{k}^{2}+\mathbb{N} \beta_{k}^{2}\right)+\mu v^{\lambda}(t)
\end{aligned}
$$

which yields

$$
v^{\lambda}(\grave{u}) \leq c+\frac{K_{a} \mathbb{N}}{1-\mu} \int_{0}^{\grave{u}} p(s) \Omega\left(v^{\lambda}(s)\right) d s
$$

Denoting by $\omega_{\lambda}^{\prime}(t)$ the right-hand side of the previous inequality, we see that

$$
\omega_{\lambda}^{\prime}(\grave{u}) \leq \frac{K a \mathbb{N}}{1-\mu}\left[p(\grave{u}) \Omega\left(\omega_{\lambda}(\grave{u})\right)\right]
$$

and subsequently, upon integrating over $[0, \grave{u}]$, we obtain

$$
\int_{c}^{\omega_{\lambda}(\grave{u})} \frac{d s}{\Omega(s)} \leq \frac{K_{a} \mathbb{N}}{1-\mu} \int_{0}^{\grave{u}} p(s) d s \leq \frac{K_{a} \mathbb{N}}{1-\mu} \int_{0}^{a} p(s) d s<\int_{c}^{\infty} \frac{d s}{\Omega(s)}
$$

This estimate permits us to conclude that the set of functions $\left\{\omega_{\lambda}: \lambda \in(0,1)\right\}$ is bounded and, in turn, that $\left\{y^{\lambda}: \lambda \in(0,1)\right\}$ is bounded in $\theta_{2}(a)$. Next we show that $\Gamma$ is completely continuous. To clarify this proof, we decompose $\Gamma$ in the form $\Gamma=\Gamma_{1}+\Gamma_{2}$, where

$$
\begin{aligned}
\Gamma_{1} y(\grave{u}) & =\int_{0}^{\grave{u}} \mathbb{S}(\grave{u}, s)\left[\theta_{1}\left(s, y_{s}+\tilde{\phi}_{s}\right)+B u(s)\right] d s, \\
\Gamma_{2} y(\grave{u}) & =\mathbb{S}(\grave{u}, 0)\left[\zeta-\theta_{2}(0, \phi)\right]-\int_{0}^{\grave{u}} \mathbb{C}(\grave{u}, s) \theta_{2}\left(s, y_{s}+\widetilde{\phi}_{s}\right) d s+\sum_{0<\grave{u}_{k}<\grave{u}} \mathbb{C}\left(\grave{u}, \grave{u}_{k}\right) I_{k}\left(y_{\grave{u}_{k}}+\tilde{\phi}_{\grave{u}_{k}}\right) \\
& +\sum_{0<\grave{u}_{k}<\grave{u}} \mathbb{S}\left(\grave{u}, \grave{u}_{k}\right) J_{k}\left(y_{\grave{u}_{k}}+\tilde{\phi}_{\grave{u}_{k}}\right), \grave{u} \in J
\end{aligned}
$$

Using the hypotheses, condition (b) and Lemma 2.1, we obtain that $\Gamma_{1}$ is continuous and 
that $\Gamma_{2}$ is completely continuous. In order to use the Ascoli-Arzela theorem we prove that $\Gamma_{1}$ takes bounded sets into relatively compact ones. As above, $B_{r}=B_{r}\left(0, \theta_{2}(a)\right)$ and $\left\|y_{\grave{u}}+\tilde{\phi}_{\grave{u}}\right\|_{\mathcal{B}} \leq K_{a} r+c_{1}=\rho$ for $\grave{u} \in J$. And also, $\|(B u)(s)\| \leq B_{0}$.

From the mean value theorem, we see that

$$
\Gamma_{1} y(\grave{u}) \in \overline{\grave{u} c o\left\{\mathbb{S}(\grave{u}, s) \theta_{1}(s, \psi): s \in[0, a],\|\psi\|_{\mathbb{B}} \leq \rho\right\}}
$$

which implies that the set $\left\{\Gamma_{1} y(\grave{u}): y \in B_{r}\left(0, \theta_{2}(a)\right)\right\}$ is relatively compact for each $\grave{u} \in J$. Moreover, from

$$
\begin{aligned}
\Gamma_{1} y(\grave{u}+h)-\Gamma_{1} y(\grave{u}) & =\int_{0}^{\grave{u}}[\mathbb{S}(\grave{u}+h, s)-\mathbb{S}(\grave{u}, s)]\left[\theta_{1}\left(s, y_{s}+\tilde{\phi}\right)+(B u)(s)\right] d s \\
& +\int_{\grave{u}}^{\grave{u}+h} \mathbb{S}(\grave{u}+h, s)\left[\theta_{1}\left(s, y_{s}+\tilde{\phi}_{s}\right)+(B u)(s)\right] d s
\end{aligned}
$$

and using that $\mathbb{S}(., s)$ verifies a Lipschitz condition, we obtain that

$$
\left\|\Gamma_{1} y(\grave{u}+h)-\Gamma_{1} y(\grave{u})\right\| \leq|h| \mathbb{N}_{1} \int_{0}^{a}\left[p(s) \Omega(\rho)+B_{0}\right] d s+\mathbb{N} \int_{\grave{u}}^{\grave{u}+h}\left[p(s) \Omega(\rho)+B_{0}\right] d s
$$

which implies that $\left\|\Gamma_{1} y(t+h)-\Gamma_{1} y(t)\right\| \rightarrow 0$ as $h \rightarrow$ 0uniformly for y $\in B_{r}(0, g(a))$. From this we infer that $\Gamma_{1} y(\grave{u}): y \in B_{r}\left(0, \theta_{2}(a)\right.$ is relatively compact in $G(a)$ and consequently that $\Gamma_{1}$ is completely continuous. This completes the proof of the assertion that the map $\Gamma$ is completely continuous.

By an application of Lemma 2.1, we conclude that there exists a fixed point $y$ of $\Gamma$. It is clear that the function $\vartheta=y+\tilde{\phi}$ is a mild solution of the system (1)-(4). This completes the proof.

\section{References}

[1] G. Arthi and K. Balachandran, Controllability of Damped Second-Order Impulsive Neutral Functional Differential Systems with Infinite Delay, Journal of Optimization Theory and Applications, (152) (2012), 799-813.

[2] G. Arthi and K. Balachandran, Controllability of second-order impulsive evolution systems with infinite delay, Nonlinear Analysis: Hybrid Systems, 11 (2014), 139153.

[3] H.O. Fattorini, Second Order Linear Differential Equations in Banach Spaces, North-Holland, Amsterdam, 1985.

[4] A. Granas, J. Dugundji, Fixed Point Theory, Springer, New York, 2003. 
[5] T. Gunasekar, M. Angayarkanni and S. Yasotha, Controllability results for impulsive neutral stochastic functional integrodifferential inclusions with infinite delay, International Journal of Pure and Applied Mathematics, 116(23), 2017, 311-326.

[6] T. Gunasekar, M. Angayarkanni and K.R. Salini, Existence and Controllability results for impulsive neutral mixed-type functional integrodifferential systems with infinite delay, Journal of Advanced Research in Dynamical and Control Systems, 10(1), 2018, 449-458.

[7] H.R. Henriquez, Existence of solutions of non-autonomous second order functional differential equations with infinite delay, Nonlinear Analysis: Theory, Methods and Applications 74 (2011) 3333-3352.

[8] Y. Hino, S. Murakami, T. Naito, Functional Differential Equations with Infinite Delay, in: Lecture Notes in Mathematics, vol. 1473, Springer, Berlin, 1991.

[9] Karthikeyan, Sundararajan and Senthil Raja D, Existence of Solutions for Impulsive Second Order Abstract Functional Neutral Differential Equation with Nonlocal Conditions and State Dependent-Delay, Research and Reports on Mathematics, 2 (1) (2018), 1-5.

[10] J. Kisynski, On cosine operator functions and one parameter group of operators, Studia Mathematica 49 (1972) 93-105.

[11] M. Kozak, A fundamental solution of a second order differential equation in a Banach space, Universitatis Iagellonicae Acta Mathematica 32 (1995) 275-289.

[12] V. Lakshmikantham, D.D. Bainov, P.S. Simeonov, Theory of Impulsive Differential Equations, World Scientific, Singapore, 1989.

[13] Y. Lin, Time-dependent perturbation theory for abstract evolution equations of second order, Studia Mathematica 130 (1998) 263-274.

[14] B.M. Miller, E.Ya. Rubinovich, Impulsive Control in Continuous and DiscreteContinuous Systems, Kluwer Academic/Plenum Publishers, New York,2003.

[15] E. Obrecht, Evolution operators for higher order abstract parabolic equations, Czechoslovak Mathematical Journal 36 (1986)210-222.

[16] F. Paul Samuel, T. Gunasekar and M. Mallika Arjunan, Controllability results for damped second-order impulsive neutral functional integro-differential system with infinite delay in Banach spaces, International Journal of Engineering Research and Development,6(2013), 21-31.

[17] A.M. Samoilenko, N.A. Perestyuk, Impulsive Differential Equations, World Scientific, Singapore, 1995. 
[18] H. Serizawa, M. Watanabe, Time-dependent perturbation for cosine families in Banach spaces, Houston Journal of Mathematics 12 (1986) 579-586.

[19] C.C. Travis, G.F. Webb, Second order differential equations in Banach space, in: Proc. Internat. Sympos. on Nonlinear Equations in Abstract Spaces, Academic Press, New York, 1987, pp. 331-361.

[20] C.C. Travis, G.F. Webb, Compactness, regularity, and uniform continuity properties of strongly continuous cosine families, Houston Journal of Mathematics 3 (1977) 555-567.

[21] C.C. Travis, G.F. Webb, Cosine families and abstract nonlinear second order differential equations, Acta Mathematica Academiae Scientiarum Hungaricae 32 (1978) 76-96. 